\title{
Association of serum lipid levels and prostate cancer severity among Hispanic Puerto Rican men
}

Jeannette Salgado-Montilla ${ }^{1}$, Marievelisse Soto Salgado ${ }^{1}$, Barbara Surillo Trautmann², Ricardo Sánchez-Ortiz ${ }^{3}$ and Margarita Irizarry-Ramírez ${ }^{2^{*}}$

\begin{abstract}
Background: While obesity and fat intake have been associated with an increased risk of prostate cancer (PCa) aggressiveness and mortality, the association between lipid levels and PCa phenotype remains unclear. Previous reports evaluating this association are inconsistent and highly variable when considering different racial/ethnic groups. There are scarce data regarding this association among Hispanics, and specifically Puerto Rico's Hispanic men, a population with a higher burden of PCa, metabolic syndrome and overweight. This population has a different ancestry profile than other Hispanics from Central and South America. Due to the above the researchers inquired if there is a relationship between serum lipid levels and PCa phenotype in this understudied population using a cohort of patients treated with radical prostatectomy as their first treatment.
\end{abstract}

Methods: We performed an exploratory retrospective medical record review study of 199 PCa patients who underwent radical prostatectomy between 2005 and 2012. Variables analyzed included age at PCa diagnosis, Body Mass Index (BMI), preoperative serum prostate-specific antigen (PSA), lipid levels, and clinical parameters such as prostatectomy pathologic stage and Gleason Score (GS). PCa severity was defined using pathologic stage and GS. Unadjusted and adjusted logistic regression models were fitted to estimate the odds ratios (ORs) with $95 \%$ confidence intervals $(\mathrm{Cl})$ to define the relationship among clinical characteristics and PCa severity.

Results: Mean age for the cohort was 58.8 years (range: 40-75), $78.9 \%$ were overweight or obese, $36.7 \%$ had hypertriglyceridemia, and $35.2 \%$ had low HDL levels. In the unadjusted logistic regression model, hypertriglyceridemia (OR: 2.11, $95 \% \mathrm{Cl}=1.13-3.93)$ ), low HDL (OR: 1.90, $95 \% \mathrm{Cl}=1.02-3.56-$ ), and age (OR: 2.34, $95 \% \mathrm{Cl} 1.25-4.40$ ) were significantly associated with a diagnosis of high severity of PCa.

Conclusions: In Puerto Rican men with PCa, elevated hypertriglyceridemia, low HDL levels, and age were statistically associated with high grade PCa on bivariate analysis. Total cholesterol level was not associated with severity of disease. Associations lost significance upon multivariate adjustment. These data generate important hypotheses regarding the potential relationship between lipid pathways and PCa development and underscore the need to perform larger scale and longitudinal studies to sort out whether, hypertriglyceridemia is associated with PCa phenotype and development.

Keywords: Triglycerides, Prostate cancer, Cholesterol, Obesity, Hispanics

\footnotetext{
* Correspondence: margarita.irizarry@upr.edu

${ }^{2}$ Graduate Department, Clinical Laboratory Sciences, School of Health

Professions, Medical Sciences Campus, University of Puerto Rico, PO Box

365067, San Juan 00936-5067, Puerto Rico

Full list of author information is available at the end of the article
} 


\section{Background}

In Puerto Rico, the age-adjusted incidence rate of prostate cancer (PCa) between 2008 and 2012 was 152.8 per 100,000 men, while the age-adjusted death rate for the same period was 30.6 per 100,000 men [1]. Of the 78 municipalities in the Island, 30 had an age-adjusted death rate of 35 or higher, which highlights a possible health disparity. Also for this period, prostate cancer was the leading cause of death by cancer in men accounting for $17.6 \%$ of all cancer deaths in men. These statistics show that PCa mortality in Puerto Rican men is higher than in Caucasians and continental United States (US) Hispanics [2-5], and warrants a study to identify the underlying causes of this high death rate.

Previous data have shown that risk factors associated with the development of PCa include age and family history. In addition a high dietary intake of animal fat or meat, and obesity seems to be related to an increase in the risk of developing the disease or its aggressiveness $[6,7]$. While studies evaluating the association between obesity and PCa have been conflicting, most suggest that obesity has a positive correlation with a higher risk of developing high-grade $\mathrm{PCa}$ [8] and dying of $\mathrm{PCa}$ [9]. In obese men, abdominal adiposity may be related to progression of existing disease and biochemical recurrence after treatment [10].

In Puerto Rico, according to statistics of the Behavioral Risk Factor Surveillance System (BRFSS) for 2012, $37.8 \%$ of citizens were overweight and $28.4 \%$ were obese [11]. The BRFSS also indicates that $39 \%$ of the population had high cholesterol levels. Moreover, a cross-sectional study evaluating the prevalence of metabolic syndrome in the metropolitan area of San Juan Puerto Rico, established that the prevalence of metabolic syndrome correlated with increasing age, rising to $58.2 \%$ in participants between the ages of 70 and 80 . Moreover, the researchers found that in their study cohort, men had higher levels in triglycerides than women [12].

Previous work by our group in 2010, established that Puerto Rican Hispanic men with a higher BMI had a higher prevalence of metastatic disease than those with a low BMI. In addition, we also reported that obese or overweight men with low levels of PSA had positive prostate biopsies, making this a population at risk for delayed diagnosis [13].

Other studies have established that cholesterol, triglycerides, and lipoproteins may play a key role in prostate oncogenesis and severity. Moses et al. in 2009 reported that increased LDL serum levels were associated with a higher risk of prostate cancer in African American men but not in non-African American males [14]. However, Adedapo and collaborators in 2012, reported that lower serum levels of triglycerides and total cholesterol were associated with benign prostate hyperplasia and prostate cancer [15]. In that same year, Hayashi et al. found that high triglycerides serum levels were positively correlated to prostate cancer incidence in a sample of Japanese men age 60 years or older. In addition, they also reported that more aggressive prostate cancer cases defined as having a GS of 8 or greater were significantly associated with triglycerides levels above $150 \mathrm{mg} / \mathrm{dL}$ [16]. In 2009, Platz, E. et al.[17] followed 5,586 men in the placebo arm of the Prostate Cancer Prevention Trial (PCPT), and reported that in a group of men with GS 8-10, those with low serum cholesterol levels had a lower risk of high grade prostate cancer with worse prognosis. Work by Solomon et al. in 2008 [18] suggests an influence of cholesterol levels on the survival of cancer cells. Moreover the relationships between cholesterol levels and high grade prostate cancer seem to be dependent on BMI, with heavy men with low circulating cholesterol showing lesser rates of high grade and advanced disease [19]. Although the above mentioned research have brought light into the possible relationship of lipids and the aetiology and phenotype of prostate cancer, there has been limited participation of Hispanics in general and Puerto Ricans in particular as part of the studied cohorts.

Considering the high prevalence of metabolic syndrome in Puerto Rico, the BMI statistics, and the health disparity in prostate cancer mortality rates in this population, it is possible that lipid levels could influence the prostate cancer phenotype in this population. We found no evidence of a previous study associating serum lipid levels with the characteristics of prostate cancer in a Hispanic Puerto Rican population. Thus, in this exploratory study we tested the hypothesis that high triglycerides and low HDL levels were associated with a more severe cancer phenotype. This work contributes data that must be considered in the clinical management of PCa patients in a population of Hispanics, with a different ancestry background than other Hispanic and racial/ ethnic groups [20].

\section{Results}

Mean age for the cohort was 58.8 years (range: 40 to 75). Overall, 99 participants (49.8\%) were $\geq 60$ years old, 157 (78.9\%) were overweight or obese, 73(36.7 \%) had hypertriglyceridemia and 70 (35.2 \%) had low HDL levels (Table 1$)$. Low severity PCa [GS $(3+4$ or less) with a low pathologic stage $(\leq \mathrm{pT} 2 \mathrm{c})$ ] was found in $140(70.4 \%)$ patients, while 59 (29.6\%) had high severity PCa.

Table 2 describes the demographic and clinical characteristics in patients by PCa severity. PCa severity varied significantly by age at cancer diagnosis, triglycerides, and HDL cholesterol levels $(p<0.10)$. In the low severity group, 97 (69.3\%) subjects had desirable HDL levels ( $\geq 40 \mathrm{mg} / \mathrm{dL}$ ) compared with 43 (30.7\%) with low HDL 
Table 1 Demographics and clinical characteristics of study sample $(n=199)$

\begin{tabular}{ll}
\hline Characteristic & $n(\%)$ \\
\hline Age at PCA diagnosis (years) & $100(50.25)$ \\
$\quad<60$ & $99(49.75)$ \\
$\geq 60$ & $58.82 \pm 6.92$ \\
Mean age \pm SD & \\
BMI (kg/m $\left.{ }^{2}\right)$ & $42(21.11)$ \\
$\quad<25.00$ & $98(49.25)$ \\
$25.00-29.99$ & $59(29.65)$ \\
$\geq 30.00$ & \\
Cholesterol (mg/dL) & $125(62.81)$ \\
$\quad<200$ & $74(37.19)$ \\
$\geq 200$ & \\
Triglycerides (mg/dL) & $126(63.32)$ \\
$<150$ & $73(36.68)$ \\
$\geq 150$ & \\
HDL Cholesterol (mg/dL) & $129(64.82)$ \\
$\geq 40$ & $70(35.18)$ \\
$<40$ & \\
LDL Cholesterol (mg/dL) & $177(88.94)$ \\
$<160$ & $22(11.06)$ \\
\hline 160 &
\end{tabular}

levels $(p=0.042)$. Low triglycerides levels were present in $96(68.46 \%)$ of the subjects in the low severity group and in $29(50.9 \%)$ of the subjects in the high severity group $(p=0.018)$. We found a higher percentage $(34 \%)$ of high severity PCA in patients with high triglycerides and $\mathrm{BMI}<30$, and only in $20 \%$ of patients with low triglycerides levels in the same BMI group. Table 3 shows the estimation of the unadjusted and adjusted OR (95\% $\mathrm{CI}$ ) and $\mathrm{PCa}$ severity. In the unadjusted regression model, a higher BMI, age $\geq 60$ years, high triglyceride levels and low HDL were significantly associated with PCa severity $(p \leq 0.10)$.

\section{Discussion}

$\mathrm{PCa}$ is a complex disease with phenotypes that appear to arise as a result of "individualized" processes in each patient. Its development may be influenced by the personal lifestyle and nutritional habits of each individual [21]. Obesity and hyperlipidemia among other components of the metabolic syndrome are known risk factors for prostate cancer [22, 23]. The Puerto Rican population has unique characteristics in terms of nutritional habits and lifestyle. Ho and colleagues showed that the traditional diet of Puerto Ricans includes a low diversity of fruits and vegetables consumption and is, therefore, "unhealthful" [24]. Colon-Lopez, V. and collaborators in 2013 [25],
Table 2 Description of demographic and clinical characteristics by severity status of $\mathrm{PCa}$

\begin{tabular}{|c|c|c|c|}
\hline \multirow[t]{3}{*}{ Characteristic } & \multicolumn{2}{|l|}{ PCa Severity } & \multirow[t]{3}{*}{$p$-value* } \\
\hline & Low $(n=140)$ & High $(n=59)$ & \\
\hline & $n(\%)$ & $n(\%)$ & \\
\hline Age at PCA diagnosis (years) & & & 0.007 \\
\hline$<60$ & $79(56.43)$ & $21(35.59)$ & \\
\hline$\geq 60$ & $61(43.57)$ & $38(64.41)$ & \\
\hline BMI $\left(\mathrm{Kg} / \mathrm{m}^{2}\right)$ & & & 0.216 \\
\hline$<25.00$ & $33(23.57)$ & $9(15.25)$ & \\
\hline $25-29.99$ & $70(50.00)$ & $28(47.46)$ & \\
\hline$\geq 30.00$ & $37(26.43)$ & $22(37.29)$ & \\
\hline Cholesterol (mg/dL) & & & 0.508 \\
\hline$<200$ & $90(64.29)$ & $35(59.32)$ & \\
\hline$\geq 200$ & $50(35.71)$ & $24(40.68)$ & \\
\hline Triglycerides (mg/dL) & & & 0.018 \\
\hline$<150$ & $96(68.57)$ & $30(50.85)$ & \\
\hline$\geq 150$ & $44(31.43)$ & $29(49.15)$ & \\
\hline HDL Cholesterol (mg/dL) & & & 0.042 \\
\hline$\geq 40$ & $97(69.29)$ & $32(54.24)$ & \\
\hline$<40$ & $43(30.71)$ & $27(45.76)$ & \\
\hline LDL Cholesterol (mg/dL) & & & 0.465 \\
\hline$<160$ & $126(90.00)$ & $51(86.44)$ & \\
\hline$\geq 160$ & $14(10.00)$ & 8 (13.56) & \\
\hline
\end{tabular}

${ }^{*} p$-value from Pearson Chi-square test. Figures in bold have statistical significance

found that in a group of 593 Puerto Ricans living in the island, only $19.5 \%$ followed the recommendations for daily exercise activity and only $4.2 \%$ met both the daily exercise and daily fruit and vegetables intake recommendations. Moreover $40.4 \%$ of the respondents reported they did not believe exercise could lower their risk of cancer.

Abdominal obesity, hypertriglyceridemia, and low HDL levels have a high prevalence among the population living on the island [26]. Puerto Rican adult males are more likely to be overweight regardless of age as reported by Perez et al. [27]. Considering these phenotypes, and previous associations of lipid levels and PCa done by others in non-Hispanic populations, [28, 29] we aimed to establish if there was relationship between serum lipids levels and prostate cancer grade in Puerto Rican men. We found that high triglycerides and low HDL levels correlate each on its own with high severity prostate cancer. Associations lost significance upon multivariate adjustments.

\section{BMI and PCa severity}

When we analyzed the association of presurgical BMI and PCa severity, our data suggest that in Puerto Rican overweight and obese men there was an increased risk for more severe disease. Nevertheless, the tendency is 
Table 3 Logistic regression models of factors associated to the PCa severity among a sample of male patients in Puerto Rico

\begin{tabular}{|c|c|c|c|c|}
\hline Characteristic & Unadjusted OR (95% Cl) & $p$-value & Adjusted OR $(95 \% \mathrm{Cl})^{\mathrm{a}}$ & $p$-value \\
\hline \multicolumn{5}{|c|}{ Age at PCa diagnosis (years) } \\
\hline$<60$ & 1.0 & & 1.0 & \\
\hline$\geq 60$ & $2.34(1.25-4.40)$ & 0.012 & $2.59(1.34-5.02)$ & 0.005 \\
\hline \multicolumn{5}{|l|}{ BMI $\left(\mathrm{Kg} / \mathrm{m}^{2}\right)$} \\
\hline$<25.00$ & 1.0 & & 1.0 & \\
\hline $25-29.99$ & $1.47(0.62-3.46)$ & 0.381 & $1.26(0.51-3.10)$ & 0.614 \\
\hline$\geq 30.00$ & $2.18(0.88-5.40)$ & 0.092 & $2.28(0.88-5.89)$ & 0.089 \\
\hline \multicolumn{5}{|c|}{ Triglycerides (mg/dL) } \\
\hline$<150$ & 1.0 & & 1.0 & \\
\hline$\geq 150$ & $2.11(1.13-3.93)$ & 0.019 & $1.78(0.91-3.49)$ & 0.093 \\
\hline \multicolumn{5}{|c|}{ HDL Cholesterol (mg/dL) } \\
\hline$\geq 40$ & 1.0 & & 1.0 & \\
\hline$<40$ & $1.90(1.02-3.56)$ & 0.044 & $1.73(0.87-3.42)$ & 0.117 \\
\hline
\end{tabular}

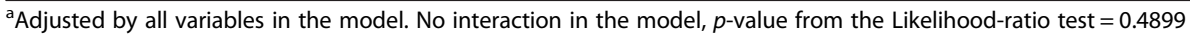

marginally significant. These results must be interpreted with caution considering the limitations imposed by using BMI as an overweight/obesity measurement. Despite its common use, BMI is not an exact measurement of body fat and does not consider body fat mass and its distribution. Therefore, it may misrepresent people with high lean body mass [30, 31]. In addition, a limitation of our study is that we do not have a longitudinal history of the patients' BMI, which hinders our ability to establish a direct relationship between high BMI and the onset and development of prostate cancer severity.

\section{Triglycerides and PCa severity}

When we analyzed the association of triglycerides and prostate cancer severity, our data suggest that in Puerto Rican prostate cancer patients with hypertriglyceridemia the risk of more severe cancer is increased. Triglycerides metabolism provides essential fatty acids [32]. It has been suggested that in prostate cancer the lack of regulation of LDL-receptor allows for the increased uptake of essential fatty acids when LDL serum levels are elevated. This provides a mechanism for Prostaglandin E2 (PGE2) synthesis, a known growth factor for cancer cells $[32,33]$. The relationship between triglycerides and the severity could be explained by the contribution that high levels of triglycerides make to the essential fatty acids available for PGE2 production mechanism.

In addition, triglycerides levels include very low density lipoproteins (VLDL), chylomicrons and remnant lipoproteins (RLP), produced after the hydrolysis of chylomicrons and VLDL [34]. These RLPs bind to the cells via the Apo $E$ and $A p o B / E$ receptors. In vitro studies have shown that $1 \mu \mathrm{g} / \mathrm{mL}$ of RLP induces the proliferation of $\mathrm{PC}-3$ prostate cancer cells, in a dose dependent manner. This dose is comparable to levels of
$10 \mathrm{ng} / \mathrm{dL}$ that have been detected in patients [35]. These results should be further characterized in an in vivo model.

\section{Total Cholesterol, HDL and PCa severity}

It is known that cholesterol plays an important role in prostate cancer as a precursor of androgens, cell proliferation mediator and inflammation [36]. Cholesterol is also included within the lipid bimolecular layer of the cell membrane, and this includes prostate cancer cells [37]. We did not find statistically significant differences in serum total cholesterol levels among the high and low severity prostate cancer. These results diverge with previous studies $[17,28]$ which finds positive correlations between lower cholesterol levels and lower risk of highgrade cancer or studies associating cholesterol with tumor growth in animal models [38-40]. It is important to notice that among the many studies done to look for associations between cholesterol levels and $\mathrm{PCa}$ or the use of cholesterol reducing drugs, ie. statins and $\mathrm{PCa}$ there are no standardized measurements for $\mathrm{PCa}$ grade or severity. Some studies use only the GS, in two categories higher or lower than 7 [32] others use three [28] and still others [17] as we do here, include the tumor stage as part of the considerations to assess the severity of the disease. Thus, the results are actually not fully comparable among the studies.

The formation of lipid rafts in PCa cells have been postulated to facilitate signaling in cancer cells that foster carcinogenic transformation throughout time. Admittedly, our cholesterol findings are limited by the lack of longitudinal data regarding the patients' cholesterol levels, thus we cannot assess the effect of the range of time that the patient had high or low cholesterol levels and the PCa phenotype. In addition, the role of other 
cholesterol susceptible pathways in carcinogenesis must be addressed.

In our study group, a higher percentage of patients with high HDL levels had low severity PCa. It has been suggested that HDL plays a protective role in the pathophysiology and cancer progression [41]. Low HDL effect could be explained by reduced binding of PON-1(paraoxonase) thus reducing PON-1free radical scavenging capacity [42]. Additionally, HDL may convey some protection from cancer severity by inhibiting the formation of lipid rafts which have been associated to procarcinogenic cell signaling through the activity of Caveolin-1(Cav-1) [43].

\section{Conclusions}

Undeniably, our study suffers from the limitations associated to a small study group. It is not a longitudinal and prospective study, thus the study design could not include an assessment of lifestyle habits that may act as confounders.

Notwithstanding the limitations imposed by sample size and lack of longitudinal data, this study sheds light into the possible effects of lipids in the PCa phenotype of an understudied population, with high overweight and PCa mortality rates. Our data was not gathered island wide but the treatment facility where we conducted our study receives patients from all over the island, thus the population, although small is not confined to the metropolitan area of San Juan. Moreover, more than $95 \%$ of the patients had some type of medical insurance, therefore we are confident that the possible effect of lack of access to medical care was probably not present in the studied group. The potential clinical implication is that, triglycerides and HDL levels may be related to PCa severity and therefore relevant to patient management, remarking that assessment of these lipid levels should be part of the routine screening and diagnostic procedures. We would like to conclude with the following: There is a need for prospective, longitudinal and well-designed studies, with adequate representation of ethnic and racial diversity to elucidate the role of HDL and LDL and total cholesterol levels, in the risk of developing PCa and in its progression.

\section{Methods}

\section{Ethics}

This study was approved by the Institutional Review Board (IRB) of the University of Puerto Rico Medical Sciences Campus (IRB approved protocol \#8860211).

\section{Study population}

The studied population was chosen from patients undergoing treatment at a private medical facility in San Juan, Puerto Rico which receives patients from all-over the island and performed 516 radical prostatectomies (RP) during the study period 2005-2012.

Inclusion criteria for the study were: Patients had to be Puerto Rican, defined as being born in Puerto Rico with parents and grandparents also from Puerto Rico, aged 40-75 years old. Importantly, Radical prostatectomy (RP) was the first treatment for the PCa. Patients were excluded if they had been diagnosed previously with other cancers or had viral infections at the time of the first screening visit. Four hundred and eighty one (481) patients' records complied with these requirements. Out of the 481, we excluded 267 records that did not have the lipid panel data. We also excluded patients records $(n=15)$ that were under statin treatment for cholesterol management. Ultimately 199 records were included in the study.

\section{Data collection procedures}

The following clinical information was recorded from medical charts: age, fasting serum lipid levels at the time of their diagnosis, pre-radical prostatectomy PSA, height, weight, and prostatectomy GS and Pathologic Stage from pathology report. Lipid data accrued included triglycerides, cholesterol, and lipoproteins (HDL and LDL). Lipid levels were recorded from the patients' first visit to the facility prior to any intervention. Lipids were categorized following the US National Institutes of Health - National Cholesterol Education Program ATP III guidelines (Table 4) [44]. Height and weight were used to calculate BMI according to Centers for Disease Control formula (weight $[\mathrm{kg}] /$ height $^{2}\left[\mathrm{~m}^{2}\right]$ ). BMI categories were defined as underweight/normal $\left(\leq 24.9 \mathrm{~kg} / \mathrm{m}^{2}\right)$, overweight $\left(25.0-29.9 \mathrm{~kg} / \mathrm{m}^{2}\right)$, and obese $\left(\geq 30.0 \mathrm{~kg} / \mathrm{m}^{2}\right)$.

Disease severity was defined using both the prostatectomy GS and the American Joint Committee on Cancer (AJCC) prostate cancer Pathologic Staging [45]. Low severity was defined as GS $\leq 7(3+4)$ and pathologic stage $\leq$ pT2c. High severity was defined as GS $\leq 7(3+4)$ and pathologic stage $\geq$ pT3a or GS $\geq 7(4+3)$ and pathologic stage $>$ pT2c.

\section{Statistical analysis}

Univariate analysis was performed to characterize the population $(n=199)$ according to demographic and clinical characteristics. Contingency tables were generated

Table 4 Lipid levels categories following the US National Institutes of Health - National Cholesterol Education Program ATP III guidelines

\begin{tabular}{lll}
\hline Triglycerides & Desirable: $<150 \mathrm{mg} / \mathrm{dL}$ & Non desirable: $>150 \mathrm{mg} / \mathrm{dL}$ \\
\hline Cholesterol & Desirable: $<200 \mathrm{mg} / \mathrm{dL}$ & Non desirable: $>200 \mathrm{mg} / \mathrm{dL}$ \\
$\mathrm{HDL}$ & Low: $<40 \mathrm{mg} / \mathrm{dL}$ & High: $>40 \mathrm{mg} / \mathrm{dL}$ \\
$\mathrm{LDL}$ & Low: $<160 \mathrm{mg} / \mathrm{dL}$ & High: $>160 \mathrm{mg} / \mathrm{dL}$ \\
\hline
\end{tabular}


to assess the relationship of demographic and clinical characteristics with prostate cancer severity using Pearson chi-square test. Unadjusted and adjusted logistic regression models were fitted to estimate the odds ratios (ORs) with $95 \%$ confidence intervals (CI) to define these relationships. Variables statistically associated with $\mathrm{PCa}$ severity $(p<0.10)$ in the unadjusted logistic regression models were included in the multivariate logistic regression model. Considering the sample size we used a level of $p \leq 0.10$ as a screening criterion for variable selection for the multivariate logistic regression model, to which a level of $p \leq 0.05$ was assigned. This statistical and epidemiological decision was based on recommendations done by Bendel and Afifi [46] and Mickey and Greenland [47] on logistic regression. These authors show that use of a more traditional level (such as 0.05) often falls to identify variables that have clinical and scientific significance. All analyses were performed using STATA 12.0 (College Station, Texas, USA)

\section{Abbreviations \\ PCa: Prostate cancer; BMI: Body mass index; PSA: Prostate-specific 1ntigen; GS: Gleason score; BRFSS: Behavioral risk factor surveillance system; IRB: Institutional Review Board; HIV: Human immunodeficiency virus; HCV: Hepatitis C virus; HDL: High density lipoproteins; LDL: Low density lipoproteins; AJCC: American Joint Committee on Cancer; OR: Odds ratios; Cl: Confidence intervals; PGE2: Prostaglandin E2; VLDL: Very low density Lipoproteins; RLP: Remnant lipoproteins; PON-1: Paraoxonase; Cav-1: Caveolin-1; $\mathrm{NIH}$ : National Institutes of Health; US: United States of America.}

\section{Competing interests}

The authors declare that they have no competing interests.

\section{Authors' contributions}

JSM: Participated in data collection and analysis and procedures in the manuscript confection. BST: Participated in data collection and analysis and procedures in the manuscript confection. MSS: Performed the statistical analysis and helped to finalize the manuscript. RSO: Participated in the study design and write up of the manuscript. MIR: Has given final approval to the manuscript. MIR is responsible for the paper contents. The manuscript represents valid work. MIR was involved in the conception design, analysis and interpretation of data and drafting the manuscript as well as obtaining funding and administrative and material support. All authors read and approved the final manuscript.

\section{Acknowledgements \\ The project described in this publication was supported by Award Number 8U54MD 007587 from the National Institute on Minority Health and Health Disparities, and by Award Grant Number\# CA096297/CA096300 from the National Cancer Institute of the National Institutes of Health. We want to acknowledge all the help of the Robotic Urology Oncology Institute. The content is solely the responsibility of the authors and does not necessarily represent the official views of the National Institutes of Health.}

\footnotetext{
Author details

'UPR/MDACC Partnership in Excellence in Cancer Research Program, Medical Sciences Campus, University of Puerto Rico, PO Box 365067, San Juan 00936-5067, Puerto Rico. ${ }^{2}$ Graduate Department, Clinical Laboratory Sciences, School of Health Professions, Medical Sciences Campus, University of Puerto Rico, PO Box 365067, San Juan 00936-5067, Puerto Rico. ${ }^{3}$ Urology Section, School of Medicine, Medical Sciences Campus, University of Puerto Rico, PO Box 365067, San Juan 00936-5067, Puerto Rico.
}

Received: 23 July 2015 Accepted: 16 August 2015

Published online: 17 September 2015

\section{References}

1. Tortolero Luna G, Zaval ZD, Pérez Ríos N, Torres Cintrón CR, Ortiz Ortiz KJ, Traverso Ortiz M, et al. Cancer in Puerto Rico, 2008-2012. Puerto Rico Central Cancer Registry. PR: San Juan; 2013.

2. Howlader N, Noone AM, Krapcho M, Garshell J, Neyman N, Altekruse SF, et al.. SEER Cancer Statistics Review, 1975-2010. In Book SEER Cancer Statistics Review, 1975-2010 (Editor ed.^eds.), based on November 2012 SEER data submission edition. City; 2013.

3. Siegel R, Naishadham D, Jemal A. Cancer statistics for Hispanics/Latinos, 2012. CA Cancer J Clin. 2012;62:283-98.

4. Siegel R, Naishadham D, Jemal A. Cancer statistics, 2012. CA Cancer J Clin. 2012;62:10-29.

5. Soto-Salgado M, Suarez E, Torres-Cintron M, Pettaway CA, Colon V, Ortiz AP. Prostate cancer incidence and mortality among Puerto Ricans: an updated analysis comparing men in Puerto Rico with US racial/ethnic groups. P R Health Sci J. 2012;31:107-13.

6. National Cancer Institute. PDQ Prostate Cancer Prevention [http:// www.cancer.gov/types/prostate/patient/prostate-prevention-pdq]. Accessed 07/23/2015

7. Willis MS, Wians FH. The role of nutrition in preventing prostate cancer: a review of the proposed mechanism of action of various dietary substances. Clin Chim Acta Int J Clin Chem. 2003;330:57-83.

8. Allott EH, Masko EM, Freedland SJ. Obesity and prostate cancer: weighing the evidence. Eur Urol. 2013;63:800-9.

9. Rodriguez C, Patel AV, Calle EE, Jacobs EJ, Chao A, Thun MJ. Body mass index, height, and prostate cancer mortality in two large cohorts of adult men in the United States. Cancer Epidemiol Biomarkers Prev. 2001;10:345-53.

10. Zilli T, Chagnon M, Van Nguyen T, Bahary JP, Guay JP, Dufresne A, et al. Influence of abdominal adiposity, waist circumference, and body mass index on clinical and pathologic findings in patients treated with radiotherapy for localized prostate cancer. Cancer. 2010;116:5650-8.

11. Behavioral Risk Factor Surveillance System Survey Data. In Book Behavioral Risk Factor Surveillance System Survey Data (Editor ed.^eds.). City: Atlanta, Georgia: U.S. Department of Health and Human Services, Centers for Disease Control and Prevention (CDC); 2012.

12. Perez CM, Guzman M, Ortiz AP, Estrella M, Valle Y, Perez N, et al. Prevalence of the metabolic syndrome in San Juan, Puerto Rico. Ethn Dis. 2008:18:434-41.

13. Negron R, Vasquez A, Nieves M, Guerrios L, Irizarry-Ramirez M. Body mass index affects the diagnosis and progression of prostate cancer in Hispanics. Ethn Dis. 2010;20(S1):168-72.

14. Moses KA, Abd TT, Goodman M, Hsiao W, Hall JA, Marshall FF, et al. Increased low density lipoprotein and increased likelihood of positive prostate biopsy in black americans. J Urol. 2009;182:2219-25.

15. Adedapo KS, Arinola OG, Shittu OB, Kareem Ol, Okolo CA, Nwobi LN Diagnostic value of lipids, total antioxidants, and trace metals in benign prostate hyperplasia and prostate cancer. Niger J Clin Pract. 2012;15:293-7.

16. Hayashi N, Matsushima M, Yamamoto T, Sasaki H, Takahashi H, Egawa S. The impact of hypertriglyceridemia on prostate cancer development in patients aged $>/=60$ years. BJU Int. 2012;109:515-9.

17. Platz EA, Till C, Goodman PJ, Parnes HL, Figg WD, Albanes D, et al. Men with Low serum cholesterol have a lower risk of high-grade prostate cancer in the placebo Arm of the prostate cancer prevention trial. Cancer Epidemiol Biomarkers Prev. 2009;18(11):2807-13. doi:10.1158/1055-9965.EPI-09-0472Men.

18. Solomon KR, Freeman MR. Do the cholesterol-lowering properties of statins affect cancer risk? Trends Endocrinol Metab. 2008;19:113-21.

19. Mondul AM, Clipp SL, Helzlsouer KJ, Platz EA. Association between plasma total cholesterol concentration and incident prostate cancer in the CLUE II cohort. Cancer Causes Control. 2010;21 (1):61-8. doi:10.1007/s10552-009-9434-8.

20. Ruano G, Duconge J, Windemuth A, Cadilla CL, Kocherla M, Villagra D, et al. Physiogenomic analysis of the Puerto Rican population. Pharmacogenomics. 2009;10:565-77.

21. Haggstrom C, Stocks T, Ulmert D, Bjorge T, Ulmer H, Hallmans G, et al. Prospective study on metabolic factors and risk of prostate cancer. Cancer. 2012;118:6199-206.

22. Chu DI, Freedland SJ. Metabolic risk factors in prostate cancer. Cancer. 2011;117:2020-3. 
23. Tande AJ, Platz EA, Folsom AR. The metabolic syndrome is associated with reduced risk of prostate cancer. Am J Epidemiol. 2006;164:1094-102.

24. Ho GY, Figueroa-Vallés NR, De La Torre-Feliciano T, et al. Cancer disparities between mainland and island Puerto Ricans. Rev Panam Salud Publica. 2009;25:394-40.

25. Colon Lopez V, Banerjee G, Gertz AM, Ortiz AP, Calo W, Finney-Rutten LJ, et al. Behavioral correlates of fruit and vegetable intake in Puerto Rico: results from the Health Information National Trends Survey. P R Health Sci J. 2013;32(4):194-9.

26. Marcial JM, Altieri PI, Banchs H, Escobales N, Crespo M. Metabolic syndrome among Puerto Ricans and other Hispanic populations. P R Health Sci J. 2011;30:145-51.

27. Perez CM, Sanchez H, Ortiz AP. Prevalence of overweight and obesity and their cardiometabolic comorbidities in Hispanic adults living in Puerto Rico. J Community Health. 2013;38:1140-6.

28. Shafique K, McLoone P, Qureshi K, Leung H, Hart C, Morrison DS. Cholesterol and the risk of grade-specific prostate cancer incidence: evidence from two large prospective cohort studies with up to 37 years' follow up. BMC Cancer. 2012;12:25. doi:10.1186/1471-2407-12-

29. Van Hemelrijck M, Garmo H, Holmberg L, Walldius G, Jungner I, Hammar N, et al. Prostate cancer risk in the Swedish AMORIS study. Cancer. 2011;117:2086-95. doi:10.1002/cncr.25758.

30. Liu P, Ma F, Lou H, Liu Y. The utility of fat mass index vs. body mass index and percentage of body fat in the screening of metabolic syndrome. BMC Public Health. 2013;13:629.

31. Chamie K, Oberfoell S, Kwan L, Labo J, Wei JT, Litwin MS. Body mass index and prostate cancer severity: do obese men harbor more aggressive disease on prostate biopsy? Urology. 2013;81:949-55.

32. Wuermli L, Joerger M, Henz S, Schmid HP, Riesen WF, Thomas G, et al. Hypertriglyceridemia as a possible risk factor for prostate cancer. Prostate Cancer Prostatic Dis. 2005:8:316-20.

33. Chen Y, Hughes-Fulford M. Human prostate cancer cells lack feedback regulation of low-density lipoprotein receptor and its regulator, SREBP2. Int J Cancer. 2001;91:41-5.

34. Havel RJ. Postprandial hyperlipidemia and remnant lipoproteins. Curr Opin Lipidol. 1994;5:102-9.

35. Sekine Y, Koike H, Nakano T, Nakajima K, Takahashi S, Suzuki K. Remnant lipoproteins induced proliferation of human prostate cancer cell, PC-3 but not LNCaP, via low density lipoprotein receptor. Cancer Epidemiol. 2009:33:16-23.

36. Pelton K, Freeman MR, Solomon KR. Cholesterol and prostate cancer. Curr Opin Pharmacol. 2012;12:751-9.

37. Morote J, Celma A, Planas J, et al. Role of serum cholesterol and statin Use in the risk of prostate cancer detection and tumor aggressiveness. Int J Mol Sci. 2014;15(8):13615-23. doi:10.3390/ijms150813615.

38. Kok DE, van Roermund JG, Aben KK, den Heijer M, Swinkels DW, Kampman E, et al. Blood lipid levels and prostate cancer risk; a cohort study. Prostate Cancer Prostatic Dis. 2011;14:340-5.

39. Mostaghel EA, Solomon KR, Pelton K, Freeman MR, Montgomery RB. Impact of circulating cholesterol levels on growth and intratumoral androgen concentration of prostate tumors. PLoS One. 2012;7, e30062.

40. Zhuang L, Kim J, Adam RM, Solomon KR, Freeman MR. Cholesterol targeting alters lipid raft composition and cell survival in prostate cancer cells and xenografts. J Clin Invest. 2005;115:959-68.

41. Kotani K, Sekine Y, Ishikawa S, Ikpot IZ, Suzuki K, Remaley AT. High-density lipoprotein and prostate cancer: an overview. J Epidemiol/Jap Epidemiol Assoc. 2013;23:313-9.

42. Eroglu M, Yilmaz N, Yalcinkaya S, Ay N, Aydin O, Sezer C. Enhanced HDL-cholesterol-associated anti-oxidant PON-1 activity in prostate cancer patients. Kaohsiung J Med Sci. 2013;29:368-73.

43. Yang G, Timme TL, Frolov A, Wheeler TM, Thompson TC. Combined c-Myc and caveolin-1 expression in human prostate carcinoma predicts prostate carcinoma progression. Cancer. 2005;103:1186-94.

44. ATP III At-A-Glance. Quick Desk Reference. In Book ATP III At-A-Glance: Quick Desk Reference (Editor ed.^eds.). City: National Institutes of Health; 2001.
45. AJCC Cancer Staging Atlas A Companion to the Seventh Editions of the AJCC Cancer Staging Manual and Handbook Second Edition. In. 2nd Edition edition: Springer; 2012: 535-546

46. Bendel RB, Afifi AA. Comparison of stopping rules in forward regression. J Am Stat Assoc. 1977;72:49-53.

47. Mickey J, Greenland S. A study of the impact of confounder selection criteria on effect estimation. Am J Epidemiol. 1989;129:125-37.

\section{Submit your next manuscript to BioMed Central and take full advantage of:}

- Convenient online submission

- Thorough peer review

- No space constraints or color figure charges

- Immediate publication on acceptance

- Inclusion in PubMed, CAS, Scopus and Google Scholar

- Research which is freely available for redistribution 